\title{
Prácticas Educativas en Contextos de Educación Pública, Inclusión Más Allá de las Contradicciones
}

\author{
Educative Practices in Public Education Contexts, Inclusion \\ Beyond Contradictions
}

\author{
Dominique Manghi * \\ Camila Saavedra \\ Nicoley Bascuñan
}

Centro de Investigación para la Educación Inclusiva, Pontificia Universidad Católica de Valparaíso, Chile

\begin{abstract}
Las políticas educativas chilenas plantean la inclusión educativa promoviendo transformaciones en las escuelas públicas (MINEDUC, 2016) para favorecer la pertenencia social y el logro de los aprendizajes (Aguerrondo, 2008), teniendo como antecedentes las políticas educativas que refieren a metas de aprendizaje y efectividad. En cada escuela pública chilena, actualmente segregada, las prácticas educativas reflejan las respuestas situadas que negocia cada institución para atender las necesidades de su comunidad frente a la demanda de educar a todos y todas, aunque las condiciones sociales son desiguales. Este estudio cualitativo, parte de un proyecto mayor (PIA CONICYT CIE 160009), busca describir, interpretar y comprender prácticas educativas de distintos actores escolares en relación con educar para la inclusión. A partir de un estudio de casos múltiple de dos escuelas municipales de la $\mathrm{V}$ región (Chile), se lleva a cabo una observación participante y diferentes tipos de registros cualitativo para analizar las prácticas que dan cuenta de cómo ha sido la transformación del sistema educativo público. Los resultados vislumbran contradicciones que conviven con respuestas educativas, las que transforman las instituciones educativas a través de prácticas concretas de distintos actores escolares. En esta complejidad de los cambios a nivel micro, nos cuestionamos los límites entre respuestas orientadas por el asistencialismo y aquellas guiadas por principios inclusivos.
\end{abstract}

Descriptores: Educación integradora; Prácticas pedagógicas; Estudiante; Profesor, Segregación escolar.

Chilean educational policies indicate educational inclusion promoting transformations in public schools (MINEDUC, 2016) to favor social belonging and the achievement of learning (Aguerrondo, 2008), having as a background educational policies that refer to learning goals and effectiveness. In each public school, currently segregated, educative practices reflect the situated answers negotiated by each institution to meet the needs of its community to face education for all, although social conditions are uneven. This qualitative study, part of a larger project (PIA CONICYT CIE160009), seeks to describe, interpret and comprehend educational practices of different school actors in relation to education for inclusion. Based on a multiple case study of two public schools of the $\mathrm{V}$ region (Chile), a participant observation and different types of qualitative records are carried out to analyze the practices as a transformation of the public education system. The results show contradictions that coexist with educational responses that transform educational institutions through concrete practices of different school actors. In this complexity of changes at the micro level, we question the limits between responses oriented by social welfare and those guided by inclusive principles.

Keywords: Inclusive education; Teaching practices; Student; Teacher; School segregation.

*Contacto: dominique.manghi@pucv.cl

ISSN: 0718-7378

www.rinace.net/rlei/
Recibido: 26/02/2018

$1^{a}$ Evaluación: 30/06/2018

Aceptado: $\quad$ 07/09/2018 


\section{Introducción}

Las últimas reformas educativas en Chile han declarado una búsqueda hacia una educación de calidad para todos. Dichos cambios deseados se tradujeron primero a escuelas efectivas, concepto que promete agregar valor al aprendizaje de sus alumnos, especialmente de aquellos de sectores de pobreza en Chile (Raczynski y Muñoz, 2007; Raczynski et al., 2013); y en los últimos años las reformas se orientan a escuelas inclusivas (MINEDUC, 2016). Ambas metas, tanto de aprendizaje eficaz e inclusión educativa para todos, se implementan solo en los establecimientos públicos.

La política educativa chilena diseñó medidas económicas dirigidas a impactar directamente y a corto plazo en lo que ocurre en el microcosmos de cada escuela, como, por ejemplo, la Ley SEP la cual inauguró en el sistema educativo la existencia de metas de aprendizaje en plazos de tiempo específicos (Raczynski et al., 2013). Es decir, se generó subvención financiera según un índice de vulnerabilidad para que la escuela dispusiera de recursos humanos y financieras con foco en lo pedagógico a través de procesos de enseñanza e implementación curricular (Weinstein, Fuenzalida y Muñoz, 2010). Estas medidas abordan el aprendizaje en un contexto de competencia y efectividad, e ignoran las condiciones reales de las escuelas y sus actores sociales, así como el tiempo que la mejora escolar como cambio cultural realmente requiere (Redondo, 2005).

En dicho contexto de educación pública y efectividad se concreta en Chile en el año 2015 la Ley 20.845 de Inclusión Escolar, la cual apunta a tres focos: no al lucro con dineros del Estado destinados a Educación, gratuidad de las escuelas que reciben subvención del Estado; y no a la selección de ingreso a dichas escuelas, con el fin de reducir la segregación escolar, explicitando un compromiso ético del Estado con una educación que favorezca la construcción de una sociedad más inclusiva (MINEDUC, 2016). No obstante esta normativa promueve un cambio estructural en el sistema educativo, no asegura por sí misma que los procesos educativos que se desarrollan en las aulas y escuelas, así como las culturas institucionales, favorezcan la inclusión. Así conviven en nuestras escuelas la urgencia de la escuela eficaz desde la cual se toman las decisiones didácticas, con aquella que recién comienza a difundirse: la escuela inclusiva.

Tal como señala Infante y otros (2013), para acercarse a estudiar las comunidades educativas definidas por el índice de vulnerabilidad como necesitadas de subvenciones especiales, más que poner atención a sus características, es importante fijarse en las acciones que promueven dichas comunidades en sus actores y en la sociedad. Las formas de habitar y convivir de los distintos actores educativos bajo el impacto de las políticas educativas, generan prácticas sociales que posibilitan dar respuestas educativas a las personas que están en la escuela. La escuela misma como institución y como campo de lucha y negociación (Ball, 2001), posibilita prácticas sociales que se acercan a lo que consideran una escuela inclusiva (Castro, 2017).

Este estudio se centra en dos establecimientos escolares públicos chilenos de la v Región y busca identificar prácticas educativas cotidianas entendidas como respuestas de la comunidad escolar en busca de coherencia con la inclusión, interpretándolas desde la complejidad de la actual educación pública y las demandas contradictorias de las políticas educativas hacia la escuela y sus agentes (López et al., 2012). A continuación, se presentan los fundamentos teóricos respecto de las prácticas educativas como respuestas a la inclusión, luego la metodología del estudio para finalmente destacar algunos aspectos 
cotidianos pero significativos a la hora de reflexionar en torno a las demandas actuales que se llevan a cabo a estas comunidades.

\section{Políticas educativas como marco para las respuestas educativas}

Una educación que no excluya se construye en las prácticas sociales que se generan en la sociedad en general y en las escuelas en particular, las cuales hasta la actualidad han sido poco valoradas para la configuración de propuestas que las resignifiquen (Castro, 2017). En este estudio concebimos las prácticas educativas escolares como una forma de actuar que refleja las decisiones de distintos actores de una escuela para dar respuesta a necesidades, situaciones o problemas del contexto educativo. Recordemos que la educación corresponde a una práctica social que no se desarrolla desligada de la política ni de la economía, sino unida indisolublemente a las prácticas en esos niveles. Las normativas, decretos y documentos legales tienen el poder performativo de regular las prácticas de la escuela, distorsionándolas desde una lógica de la competencia y la rendición de cuentas (Ball, 2001). Las prácticas educativas entre profesores y estudiantes se construyen en la vida cotidiana, pero a la vez desde la organización económica, política y social de la que formamos parte (Rivero, 2013).

Para poder comprender las respuestas de los colegios que buscan mantener el foco en lo educativo, es necesario considerar dos niveles: el primero, situar las prácticas en un macrocosmos educativo, comprender cómo las prácticas educativas se desarrollan en el marco de las políticas públicas impulsadas por el Estado para la educación pública. El segundo nivel se relaciona con el microcosmos escolar, respecto de cómo las prácticas educativas hacen frente a la inclusión/exclusión en la vida cotidiana de la escuela pública, la cual se encuentra en un entramado situado, de lo que en nuestro país se concibiría como educación pública (Duschatzky y Birgin, 2001).

En relación al nivel macro, las políticas educativas chilenas de los últimos 30 años se caracterizan por fortalecer la privatización en desmedro de la educación pública (Redondo, 2005). Para comprender la complejidad de las políticas educativas en el contexto chileno $\mathrm{y}$ sus repercusiones en contextos educativos concretos, es necesario considerar dos aspectos.

El primer aspecto hace referencia a las características de las escuelas públicas chilenas, las que derivan del proceso de Municipalización llevado a cabo durante el mandato de Augusto Pinochet. En ese momento y bajo los principios de descentralización y libertad de enseñanza, se instauraron las 3 modalidades de financiamiento y administración de la educación: 1) municipal: aquellas financiadas por el Estado y administradas por las municipalidades; 2) particular: aquel proyecto privado financiado en su totalidad por cada familia; y 3) particular subvencionada: aquellos proyectos privados que cuenta con aportes del Estado. Dicha segregación se ha profundizado en el tiempo (Moreno-Doña y Gamboa, 2014).

Las reforman más recientes buscan revertir la situación de las escuelas públicas con foco hacia la calidad y equidad. El giro hacia la calidad educativa como medición y rendición de cuentas se materializa el año 2009 con la Ley General de Educación (LGE) que sienta las bases para el rol subsidario de Estado y un Sistema de Aseguramiento de la Calidad. Posteriormente y de manera segmentada, para compensar la calidad de la educación de los 
sectores más pobres se promulga, por una parte, la Ley de Subvención Escolar Preferencial (SEP) que provee de nuevos recursos financieros y profesionales a las escuelas (Weinstein, Fuenzalida y Muñoz, 2010). Por otra parte, se suma en el año 2015 la Ley de Inclusión Escolar que busca un cambio estructural poniendo fin al lucro de las escuelas financiadas con dineros estatales, obligando a no seleccionar a sus estudiantes, y a ser gratuitas. Identificamos acá una primera contradicción, mientras una persigue una escuela eficaz estableciendo contextos de competitividad y midiendo vulnerabilidad, la otra busca transformar las mismas escuelas competitivas en comunidades inclusivas, con la promesa de que en el interior de la escuela se revertirá la desigualdad socio económica.

La actual conformación de las escuelas chilenas, especialmente en contextos urbanos, da cuenta de comunidades segregadas, pero homogéneas en su interior en cuanto al origen social de estudiantes y sus familias (Mizala y Torche, 2012) producto del sistema económico que ha fomentado un mecanismo de selección social. Cuando el foco se pone en contextos de riesgo social y pobreza, se apunta principalmente a aquellas escuelas que reciben subvención estatal y se identifican con un alto índice de vulnerabilidad (Infante et al., 2013) en las cuales además es frecuente el abandono y repetición escolar (Aguerrondo, 2008). De esta manera, identificamos una segunda contradicción en el sistema educativo, la vulnerabilidad traducida a un número se ha instalado como un sistema de razonamiento, reforzando la selección social y la segregación (Infante et al., 2013).

Para dimensionar los efectos en educación pública, cabe mencionar dos particularidades del sistema chileno. Por una parte, las políticas públicas solo apuntan a los colegios que reciben subvención del Estado: principalmente los municipales y luego los particulares subvencionados. Esto nos señala que la forma de pensar la educación del país, no incluye a aquellas instituciones privadas, que mantienen el privilegio del principio de libertad de enseñanza. Por otra parte, la aplicación de un conjunto de políticas educativas solo a los colegios considerados parte de la educación pública implica además que las evidencias de logro de metas de desempeño y el énfasis en rendición de cuentas del colegio respecto de las múltiples subvenciones estatales, vienen por añadidura justamente a este grupo de escuelas, distorsionando las prácticas educativas que allí se desarrollan (Ball, 2001) agregando una contradicción más a la lista.

El segundo aspecto se refiere a las políticas internacionales para una educación inclusiva. Los organismos internacionales como UNESCO y OCDE han iniciado desde fines del siglo pasado un movimiento para la inclusión en educación. En el año 1990 representantes de 155 países declaraban el énfasis en la Educación Para Todos en la conferencia mundial de Educación en Jomtiem (UNESCO, 1990), reconociendo la educación como un derecho humano y se proponía en un plazo de 10 años universalizar el acceso al aprendizaje con foco en la educación primaria, y fomentar la equidad. Cumplido el plazo, pero no dichas metas, la OCDE (2012) mantiene estos propósitos afirmando que la equidad en educación puede revertir el efecto de inequidades sociales y económicas, idea que funciona como una promesa social. Para esto propone abordar dos dimensiones: un trato justo para todos, lo que significa que circunstancias sociales y personales no deberían ser obstáculos para logros educativos; y una educación inclusiva, la cual se lograría si todos alcanzan estándares mínimos de educación.

Ambos organismos coinciden en que para concretar la agenda inclusiva convergen dos aspectos: un aspecto social en relación a equipar oportunidades de desarrollo de las personas independiente de sus circunstancias económicas, sociales o culturales; y otro 
aspecto referido a que dicho desarrollo y logro educativo debe verse reflejado en aprendizajes mínimos. Es lo que Murillo (2003) ha denominado "eficacia de la mejora escolar" o Ainscow (2005) "mejora con actitud", en los cuales se entiende la inclusión en términos de metas de aprendizaje, es decir escuelas eficaces e inclusivas simultáneamente. Esta doble preocupación busca transformaciones profundas y sistémicas de las escuelas, sus aulas y relaciones pedagógicas, así como del currículo y la enseñanza para que la educación inclusiva no sea solo un sueño, sino que se plasme en prácticas educativas concretas (Echeita et al., 2014; Escudero y Martínez, 2012).

En la actualidad aún siguen vigentes tres formas de marginación educativa especialmente en la educación pública: marginación por exclusión total que se refiere al acceso al sistema educativo; marginación por exclusión temprana que implica abandono escolar en los primeros niveles; y marginación por inclusión que se produce por la segmentación del servicio educativo en circuitos de diferente calidad. Esta última nos indica que los estudiantes en escuelas públicas, pese a que están "incluidos" en el sistema educativo son excluidos de su participación social y sus potenciales aprendizajes curriculares (Aguerrondo, 2008). Esta situación implica que muchos estudiantes acceden y permanecen en el sistema escolar sin garantizar el desarrollo de las habilidades básicas. La marginación se explicaría según la OCDE (2004), porque la educación está determinada por tradiciones sociales, las que en Chile han sido conservadoras y han reproducido un pasado de desigualdad social: "la educación chilena está influenciada por una ideología que da una importancia indebida a los mecanismos de mercado para mejorar la enseñanza y el aprendizaje" (OCDE, 2004, p. 290). En el informe del año 2017, la OCDE reconoce que los estudiantes con mal desempeño escolar o aquellos identificados como indisciplinados o con mala conducta siguen teniendo un potencial riesgo de discriminación y posibilidad de exclusión del sistema escolar.

Para responder al diagnóstico y planteamientos de estos organismos públicos, los sistemas educativos latinoamericanos han desarrollado cuatro tipos de estrategias (Aguerrondo, 2008). Las tres primeras estrategias: asistencialismo, psicopedagogismo y las políticas compensatorias no cuestionan el sistema educativo, sino que lo mantienen. Solo la cuarta: la inclusión, propone cambiar el modelo para superar la exclusión.

La primera estrategia es el asistencialismo, busca responder a la desigualdad social presente en los sectores más vulnerables apuntando a sus necesidades básicas mediante la provisión de elementos materiales necesarios para su educación: alimentación, útiles, prendas de vestir y atenciones médicas. Una segunda estrategia es el psicopedagogismo, el cual frente a evidencias de escaso aprendizaje de la educación pública recurre a otros profesionales como psicopedagogos y psicólogos para atender el fracaso atribuido al alumno, entregando un "tratamiento" a sus necesidades. La tercera estrategia, corresponde a políticas compensatorias mediante las cuales se entregan más herramientas a los profesionales para enfrentar la enseñanza, reconociendo que la estructura institucional y la propuesta pedagógica requieren apoyos.

En estas tres estrategias mencionadas, la educación inclusiva se mal entiende, puesto que mantiene formas de segregación sutiles a grupos minoritarios en aulas ordinarias (Slee, 2012) fomentando, por una parte, la cultura escolar de la clasificación de estudiantes de acuerdo a sus características, ritmos, estilos, comportamiento, etc., siempre en contraste con parámetros de normalidad. Así es común escuchar hablar del estudiante "especial", "diferencial", "integrado" (Castro, 2017). Por otra parte, la competencia predomina dentro 
de las aulas en un sistema que busca metas de aprendizaje en vez de formar ciudadanos para la sociedad (Slee, 2013). Esto refuerza la idea de "la normalidad" de algunos, entendida como un centro donde se encuentran los que aprendieron de forma "normal" en un plazo "normal", lo que genera un circuito de exclusión (Graham y Slee, 2008).

Solo la cuarta estrategia: la inclusión, aborda la necesidad de cambios más profundos en el sistema educativo desde dos perspectivas; por una parte, retrospectiva que se relaciona a que la educación debe ofrecer igualdad de oportunidades de acceso a la escuela; y, por otra parte, prospectiva, que implica que la equidad es dar a cada uno según sus necesidades (Aguerrondo, 2008).

En relación al segundo nivel, las prácticas en el microcosmos escolar, los estudios sostienen que la escuela como institución de educación formal tiene una tradición de ser un espacio homogeneizador, en el cual se reproducen los conocimientos, saberes y valores considerados como fundamentales para dicha cultura y dignos de ser transmitidos a las nuevas generaciones, invisibilizando los rasgos particulares de los estudiantes (Castro, 2017).

Dada esta tradición, la transformación de la escuela se hace urgente si realmente interesa avanzar hacia la inclusión, y no solamente hacer cambios superficiales que maquillen lo que ocurre en las escuelas, manteniendo el statu quo (Graham y Slee, 2008).

Mientras en las escuelas, las personas -todos los agentes de la comunidad educativavivencian los efectos de las políticas públicas, al mismo tiempo intentan dar respuestas coherentes a las demandas sociales en un contexto de eficacia y competencia, a lo que se agrega la segregación socioeconómica y la marginación por inclusión (Sinisi, 2013). Entre las estrategias internacionales para transformar el microcosmos escolar, se encuentra el libro el Índice de Inclusión que es difundido en nuestro país a través de la página del Ministerio de Educación. En su primera versión, el Índice afirma alinearse con la declaración de Salamanca para que la escuela acoja a todos los estudiantes sin importar su condición (UNESCO, 1994) enfocándose en la mejora de la escuela con el fin de fomentar la participación y así el aprendizaje de todos los estudiantes (Booth y Ainscow, 2002). Para lograr aquello, presenta preguntas desde una perspectiva contextual, basada en investigación acción en escuelas inglesas, e intenta eliminar el foco en las necesidades educativas especiales, instalando en su lugar los conceptos contextuales de barreras y facilitadores, reconociendo la convivencia de normativas contradictorias que mantienen lógicas de clasificación y segregación al interior de la escuela (Manghi y Aranda, 2017).

Los autores complejizan la definición de inclusión planteándola como un "proceso de desarrollo que no tiene fin, ya que siempre pueden surgir nuevas barreras que limiten el aprendizaje y la participación, o que excluyan y discriminen de diferentes maneras a los estudiantes“" (Booth y Ainscow, 2002, p. 8)

Ahora bien, en su nueva edición del año 2011 en inglés, el Índice de Inclusión se ha centrado en el concepto de valores inclusivos, dejando de reconocer las tensiones entre políticas incoherentes para plantear un tronco común inclusivo en los diferentes programas y proyectos que ya conviven en una escuela (Manghi y Aranda, 2017).

El enfoque del Índice de Inclusión comprende el aprendizaje como una forma de pertenecer a las comunidades y es coherente con la mirada antropológica y sociocultural del aprendizaje situado de Lave y Wenger (1991). Los autores proponen que aprender es más que un proceso que ocurre en la mente de manera individual, sino más bien tiene que 
ver con que la comunidad reconozca al aprendiz como legítimo, asumiendo que los aprendizajes no se dan fuera de los valores y actitudes de quienes son los más veteranos de la comunidad que pueden favorecer u obstaculizar la incorporación de los nuevos en los grupos sociales. Los más antiguos serían los encargados de mantener la cohesión y estatus quo de la comunidad. Si reconocemos estos valores implícitos como un curriculum oculto siempre presente en cada comunidad educativa, desde la pedagogía nos preguntamos qué enseñamos cuando segregamos, excluimos o deslegitimamos a nuestros aprendices (Manghi, 2017).

En Chile, el tema de la inclusión se abordó inicialmente de manera estrecha desde la educación especial como un paso siguiente al paradigma de la integración (Opazo, 2013) y con foco en la discapacidad. Para ampliar la educación inclusiva como una meta de la escuela, el Ministerio de Educación propuso orientaciones para la creación de comunidades inclusivas (2016) mediante un documento alineado con la primera versión del Índice. En este plantean dos grandes ideas: define la inclusión en oposición a la integración trasladando la responsabilidad desde el individuo a la comunidad escolar, planteando actividades de reflexión en relación a la diversidad centrado principalmente en los profesores del colegio. La otra idea enfatiza dos temas a reflexionar de manera implícita: educación intercultural graficándolo con imágenes de escolares migrantes y de pueblos originarios; incluyendo además el tema de diversidad sexual mostrando familias homoparentales (Manghi y Baeza, 2017).

Más allá de los documentos, al interior de las comunidades la inclusión se desarrolla como un proceso de negociación (Ball, 2001) que busca dar sentido a las políticas públicas nacionales de manera local y situada, para que aporten concretamente a mejorar las condiciones de todos, pero especialmente de aquellos que pertenecen a poblaciones desfavorecidas, a través de condiciones coherentes para los estudiantes y para el trabajo docente. No obstante, muchas veces a los estudiantes que presentan situaciones de vida desfavorables, las condiciones escolares también les son duras (Baquero, 2013). El sistema escolar asume esta situación como fracaso escolar, lo cual desde una perspectiva inclusiva conlleva a cuestionar ya no el fracaso del estudiante sino el de la escuela (Terigi, 2009). Todos quienes pertenecen a la comunidad escolar son responsables de lidiar con sus prejuicios frente a las situaciones contextuales con que se presentan sus educandos, ya que estas influyen en sus rendimientos y su desarrollo personal (Baquero, 2013).

De esta manera, la investigación en educación inclusiva debe colaborar en develar las políticas y prácticas que excluyen a las minorías, y estas no son solo la presencia de una población con necesidades de apoyo, si no que de manera importante la población en situación de vulnerabilidad socioeconómica en la educación pública (Artiles y Kozleski, 2016). Es necesario tener presente las estructuras, políticas y prácticas de educación, para mirar la educación inclusiva en base a valores que atiendan a la comunidad, al reconocimiento y la representación de la diferencia, en función de conseguir un cambio social (Slee, 2013).

\section{Método}

Este estudio es parte del proyecto "Prácticas de aula y de Escuela para la inclusión" CIE 160009, y de la tesis desarrollada por Bascuñan y otros (2017). La investigación se posiciona en un paradigma cualitativo para describir, interpretar y comprender las 
prácticas educativas de distintos agentes escolares como respuestas de la comunidad escolar en busca de coherencia con la inclusión y la transformación de la escuela. A partir de un estudio de casos múltiple correspondiente a dos escuelas municipales de la comuna de Viña del Mar, se recogió información desde un enfoque etnográfico, a través de la observación participante y de registros escrito y fotográfico.

Los estudios de caso etnográficos nos permiten mantener la complejidad de "lo real" reconstruyendo prácticas y sentidos de quienes protagonizan la vida cotidiana escolar y, a la vez, abrir la posibilidad de situar dichas prácticas y sentidos en las estructuras sociohistóricas de las que forman parte (Sinisi, 2013), como por ejemplo, las políticas públicas en educación que configuran a los actores de las escuelas y su experiencias sociales e históricas, conformando tramas sociales más amplias (Achilli, 2013).

A través de la perspectiva metodológica cuasi-etnografía sociotécnica (Awasthy, 2015) se observó cada comunidad durante 12 jornadas, tanto en los espacios compartidos como patios así como en un aula en particular, en ambos casos un tercero básico. La información recolectada se analizó en función de la comprensión de cada estudio de caso, levantando categorías representativas de las prácticas de cada escuela a partir del proceso de comparación y contraste (Strauss y Corbin, 2002). A continuación se presentan algunos ejemplos seleccionados en base a las categorías de negociación y de contradicción preguntándonos que aprenden los estudiantes frente a estas prácticas, para interpretar estas prácticas como aquellas que dan espacio a la transformación de la escuela.

\section{Resultados}

Los ejemplos seleccionados en este apartado grafican las permanentes negociaciones que ocurren en los espacios escolares, bajo la premisa de que impactan en la formación de las identidades de los aprendices. Los distintos actores escolares adultos, más allá de los profesores, co-construyen junto con los estudiantes espacios que delimitan quienes pertenecen a ellos y quienes no, legitimando a quienes consideran aprendices en la escuela. Esto se vuelve más relevante aun, cuando hablamos de contextos donde los derechos básicos de las personas han sido vulnerados. Nos referiremos en este apartado a la condición de vulnerabilidad no como el resultado de un índice estadístico para asignar subvención, sino como el reconocimiento de la vulneración de derechos, definición desde la cual proponemos algunas reflexiones.

\subsection{Negociar en el microcosmos: identidad de aprendiz y espacios de pertenencia}

El primer estudio de caso del cual rescatamos los ejemplos corresponde a un colegio caracterizado por educar a varias generaciones de familias asentadas en terrenos tomados en los cerros de Viña del Mar, cerca de las zonas turísticas, pero al mismo tiempo lejos de la actividad de turismo típica de esta ciudad (Jorquera y colaboradores, 2017). La responsabilidad social de transformar las proyecciones de vida de estos estudiantes se traslada desde las políticas generales hacia la realidad concreta al interior de cada aula. Es aquí donde podemos observar las prácticas educativas con las cuales los distintos actores intentan responder a las condiciones de desigualdad social de sus estudiantes para potenciar sus aprendizajes.

Este mismo colegio es un lugar de encuentro de múltiples formas de convivencia, y es en estas formas de comunicarse con otros, de reaccionar a lo que dicen y hacen los pares y los adultos es que los escolares construyen la identidad de ser aprendiz (Julio, 2017). Dado 
que conceptualizamos los procesos de inclusión como negociación entre los distintos actores escolares (Grech, 2017), revisaremos algunas prácticas referidas a la negociación, unas en relación al uso de los espacios dentro de la escuela y la otra en cuanto a los estilos de regulación de conductas dentro del aula.

Respecto de la primera negociación, se relaciona con el uso de los espacios educativos. En esta escuela, la cual presenta una infraestructura antigua de pabellones combinado con salas en containers metálicos, se observó la conformación de un espacio distinto al esperado en el Centro de Recursos para el Aprendizaje, empleado tanto por estudiantes como docentes.

Desde la normativa ministerial, el Centro de Recursos para el Aprendizaje (CRA) en Chile busca alejarse de una biblioteca tradicional, para ser un lugar acogedor con material audiovisual y recursos interactivos para favorecer a la enseñanza y el aprendizaje (MINEDUC, 2014). En esta escuela observamos que la comunidad le da otro uso, ingresando estudiantes a diferentes horas, no solo para leer o consultar información en los computadores, sino también para escuchar música y conversar, creando un clima diferente al esperado. Esto ha provocado que el encargado del CRA, directivos y profesores flexibilicen el uso original de este lugar.

En la actualidad, el CRA en este colegio destaca como un espacio donde los jóvenes se señalan sentirse acogidos, alejándose del ambiente de una biblioteca en torno a la práctica exclusiva de lectura, para acoger prácticas de comunicación y expresión de diversa índole. Los estudiantes pueden desenvolverse en este lugar sin ningún tipo de límites frente al volumen en el que conversan, o el tipo de música que escuchan. Si miramos el CRA desde una perspectiva inclusiva restringida, observamos a primera vista que es un espacio que no favorece la accesibilidad a los estudiantes con movilidad reducida de la escuela, quienes se desplazan en silla de ruedas. No obstante, desde una perspectiva amplia y transformadora del microcosmos escolar, pese a las dificultades de accesibilidad física al mismo y de la extensión reducida de esta sala, el acceso se soluciona involucrando a los compañeros para facilitar que todos puedan ingresar y salir del espacio, y la aglomeración de quienes concurren a este espacio no parece ser considerada negativa. Por el contrario, el CRA se muestra como un espacio comunitario, donde los estudiantes disfrutan estar, sin importar edad o nivel escolar y compartir con sus pares, profesores y otros actores educativos, siendo un lugar que convoca a la comunidad educativa.

Reconocemos que el equipo directivo del colegio acoja este tipo de necesidad, como una respuesta educativa en busca de coherencia con la inclusión. ¿Qué aprenden los estudiantes frente a estos cambios en el uso de los espacios de su escuela? Antes de responder, más vale volver a pensar de manera situada las prácticas escolares en el contexto sociocultural y ante las exigencias de la política en educación. Más allá de la exigencia legal de que la escuela cuente con rampas, accesos universales e infraestructura adecuada para el quehacer educativo y para la creación de comunidades inclusivas -que aquí a todas luces no se cumple-, es necesario resituar la escuela en un entorno donde las viviendas y espacios de encuentro social han sido levantados por los propios habitantes del lugar, con escases de recursos y al margen de los servicios básicos, y donde el sitio de encuentro es la calle, la esquina, entre disparos cotidianos y tráfico de drogas. En este contexto, cuando existe un espacio dentro de la escuela que se transforma para ellos, los estudiantes aprenden que la escuela está atenta a sus necesidades, conoce sus gustos e intereses, y se 
preocupa por generar un espacio para expresarse, recogiendo la cultura de la comunidad para valorarla dentro de la escuela.

Este cambio requirió una negociación, ya que el CRA permite las conversaciones y la música, pero a la vez el volumen y forma de comunicarse no es la misma que ocurre en la calle y vela por la convivencia de las distintas personas que concurren a este espacio. Aprenden que la escuela los legitima como aprendices.

En cuanto a la segunda negociación en este microcosmos escolar, debemos recordar que los docentes se encuentran en un lugar de poder frente a su grupo de estudiantes (Godoy y Córdova, 2017). Es desde ese lugar como autoridad, que regulan de diferente manera las conductas de sus estudiantes negociando formas de participación en las actividades que proponen. Los estudiantes del $3^{\circ}$ básico observado asisten a clases, los profesores saben que sus actividades compiten con lo que ocurre en las calles y que es muy atractivo para los estudiantes (Jorquera et al., 2017). De esta manera, sus decisiones impactan en el desarrollo emocional y modelan formas de relacionarse con otros, ya sea de manera positiva o negativa.

El aula como un espacio de encuentro cultural (Julio et al., 2016), es un microcosmos donde habitan diferentes formas de comportarse, de comunicarse, de convivir. ¿Cómo puede un profesor en este contexto educativo insistir en que los estudiantes realicen las actividades propuestas? ¿Cómo negocia que sus aprendices participen en lo que les propone? La manera en que los profesores eligen hacer esta regulación influencia no sólo a quien dirigen específicamente algún comentario, sino también a sus compañeros, quienes aprenden a partir de este modelo de relacionarse con otros. Describiremos tres ejemplos de un $3^{\circ}$ básico, para luego interpretarlos desde la inclusión como transformación de la escuela.

Un primer ejemplo en relación al estilo de regulación se observa cuando un profesor al frente de todos los compañeros de curso, pide en voz alta a Francisca que se siente y ella no se sienta. Luego todo el curso se siente autorizado a gritar “FFrancisca!”, sin que el profesor tenga una postura frente a esta intervención del grupo. Ante esto -gritos de sus compañeras y silencio del profesor- ella se sienta y son los propios compañeros quienes la califican: "Es más porfiada", tal como lo haría un adulto, asumiendo la autoridad del profesor. El profesor vuelve a permanecer en silencio, ignorando esta situación.

El segundo ejemplo corresponde a un profesor frente al curso bullicioso, quien constantemente se dirige a los estudiantes para intentar apaciguarlos, amenazando con citar a los padres o con quitar el recreo. Estas estrategias son utilizadas como un medio de mantener un clima silencioso en el aula. Este mismo docente muy frecuentemente durante sus clases utiliza llamadas de atención a algunos estudiantes con un volumen muy elevado, interfiriendo en el clima que él mismo busca construir para aprender. Es más, cuando realizan mal la actividad, señala que es debido a su falta de atención a las instrucciones entregadas como la causa de la tarea desarrollada de una manera que no cumple sus expectativas. Este curso generalmente se involucra con las actividades que les plantean los profesores, sin embargo, se observa que la participación de los estudiantes disminuye durante las clases de este profesor.

Una tercera forma de regular el comportamiento en el aula la observamos de un tercer profesor frente al mismo grupo de estudiantes. Cuando ellos se encuentran conversando mientras el profesor explica algún tema al grupo, el profesor opta por llamar la atención 
identificándolos por el nombre y haciendo preguntas acerca de lo que está enseñando. El profesor no pide específicamente que guarden silencio ni ofrece algún castigo, sino que los devuelve al tema curricular, logrando que los estudiantes vuelven a poner atención a su explicación.

Consideramos estos tres estilos de regulación de la conducta como prácticas cotidianas que funcionan como respuestas educativas dentro del aula a situaciones rutinarias. ¿Qué enseñan estas formas de llamar la atención a los estudiantes? ¿qué aprenden los jóvenes de estos profesores, como adultos que modelan formas de relación social? ¿cómo se transforma la escuela de manera coherente con la inclusión?

En la negociación en el aula unos aprenden que gritar y descalificar a sus pares es lo que se espera en el aula, como una forma de colaborar con el profesor que acepta esta forma de relacionarse para lograr entre todos un clima propicio para el aprendizaje a través de llamados permanentes de atención y descalificaciones. Otras veces aprenden que el uso naturalizado de la amenaza como recurso para la regulación del comportamiento de los estudiantes es parte de la rutina cotidiana para lograr el aprendizaje. Aprenden que el profesor es quien tiene la razón, y ellos culpables de no comprender las instrucciones para ser eficientes en resolver las tareas escolares. Mientras en otros momentos aprenden que el adulto conoce sus nombres y le interesa saber qué han entendido de lo que les está proponiendo atender. En todos los ejemplos, lo que aprenden los estudiantes es lo que se espera de ellos como aprendices, es lo que algunos llaman el oficio de ser estudiante (Dávila, Ghiardo y Medrano, 2005).

En estas aulas, la clase se mantiene silenciosa por momentos, pero eso no asegura participación ni aprendizaje sino no se comparten valores inclusivos a la base (Ainscow y Booth, 2015). La inclusión no es un fin en sí mismo sino un principio para desarrollar espacios democráticos (Slee y Allan, 2011), y estas interacciones dan cuenta de profesores que desean ser escuchados, que necesitan que sus estudiantes presten atención a lo que les explican, ya que incluso son evaluados profesionalmente por esto. En estos casos, las estrategias para lograr un clima que cada profesor considera propicio para aprender, nos indican que responden a este requisito de distinta manera, no necesariamente de manera coherente con la inclusión. Algunos profesores parecen sumarse a una indiferencia colectiva, en la cual prefieren mirar hacia otro lado e ignorar conflictos que podrían ser abordados desde una perspectiva pedagógica (Slee, 2013). Si la escuela es el lugar del que disponen los estudiantes para conocer otros modelos de interacción en los que se propicie el diálogo, la argumentación ciudadana, modelos diferentes a los vernáculos que aprenden en casa y en sus barrios, en muchos casos estamos desperdiciando esta oportunidad.

A continuación revisaremos ejemplos del segundo estudio de caso, estos se relacionan con las decisiones de los profesores respecto de cómo promover el aprendizaje del currículo, ofreciendo respuestas educativas. Esta es una pequeña escuela de educación básica, que atiende a estudiantes de distintos sectores de la parte alta de Viña del Mar, de alta vulnerabilidad social. Es una escuela que se ha enfocado en avanzar en mediciones estandarizadas como un reflejo de niveles de logro de aprendizaje curricular, superando sus propios logros anteriores en pruebas nacionales como el Simce.

En este contexto, revisaremos prácticas de trabajo colaborativo entre pares, y luego opciones de los profesores respecto del tipo de interacción que promueven las diferentes actividades. 
En primer lugar, revisaremos ejemplos de prácticas de trabajo colaborativo entre pares y luego, reconocemos las prácticas pedagógicas y didácticas más frecuentes en esta aula. En esta sala de clases de $3^{\circ}$ básico se ha optado por una disposición espacial del mobiliario de la sala diferente al tradicional. La disposición de los muebles en una U alrededor de la profesora, y enfrentando el pizarrón, señala que se persigue el trabajo colaborativo y el aprendizaje innovador, ya que las mesas de trabajo donde los estudiantes se ven de frente entre ellos y la profesora, promueve otras formas de interacción y de aprendizaje.

Respecto de las primeras, las de trabajo colaborativo, se dan de manera espontánea entre los estudiantes. Por una parte, hay dos estudiantes quienes destacan por su buen rendimiento, realizan las actividades de manera rápida, terminando mucho antes que sus compañeros. Al poner atención a la rutina diaria en esta aula, observamos que estos dos niños ayudan a quienes van a un ritmo más lento, desplazándose hasta sus escritorios, acompañándolos en el desarrollo de tareas. Los estudiantes eligen a quien acompañar en su aprendizaje, y orientan el desarrollo de las tareas, repitiendo instrucciones y resolviendo dudas; constituyen un modelo sobre cómo enfrentar y relacionarse con el resto de los compañeros desde las capacidades que cada uno está desarrollando.

Los profesores a cargo van rotando según los horarios de asignatura, ninguno les impide moverse por la sala y apoyar a sus compañeros, ni tampoco promueve esta práctica entre pares. Esto evidencia que algunos estudiantes consideran que es importante aprender de manera colaborativa, sin discriminar, ayudando a los compañeros. Los profesores parecen no reconocer esta práctica colaborativa entre pares, siendo indiferente a esta interacción sin desarrollarla ni facilitarla, ni tampoco valorarla positivamente.

Por otra parte, pese a la disposición del mobiliario, hay casos en que el comportamiento de algunos estudiantes es sancionado, dejándolos fuera de las mesas en U. De esta manera, uno o más estudiantes deben sentarse apartados del resto de sus compañeros. Uno de ellos menciona reiteradas veces durante la clase que no le gustaba trabajar en aquel lugar, porque no escucha bien las instrucciones y se desconcentra en las tareas.

¿Qué aprenden los estudiantes de estas prácticas? Aprenden que en el aula los profesores no promueven ni censuran que se ayuden entre ellos, no es algo que interese desarrollar. A la vez aprenden que podemos dejar algunos compañeros fuera de las actividades, sin legitimarlo como aprendiz, eso es parte del acontecer normal de la rutina escolar.

El segundo grupo de ejemplos se relaciona con la manera en que los profesores ofrecen oportunidades de aprendizaje a sus estudiantes de $3^{\circ}$ básico. Lo más frecuente se concentra en dos tipos de práctica: trabajo con guía de aprendizaje y con dictado. Respecto de la primera práctica, los profesores desarrollan el trabajo del contenido curricular mayormente a través de guías de aprendizaje que solicitan respuestas de manera escrita. En este tipo de actividades, el profesor da las instrucciones iniciales y el resto del trabajo es individual: los estudiantes leen preguntas, buscan información escrita y escriben sus respuestas. Aquellos estudiantes que presentan dificultades en la asignatura, parecen no atender ni comprender las instrucciones, por lo que se observan distraídos y conversando de otros temas durante las actividades, sin llegar a escribir sus respuestas.

Respecto de la otra forma frecuente de desarrollar las clases es a través del dictado, los profesores enseñan dictando en voz alta, con lo que aseguran el silencio en clases. Los estudiantes intentan no perder lo dictado, manteniéndose atentos para transcribir en sus cuadernos. 
Recordemos que este es un $3^{\circ}$ básico, algunos de los niños aún están afianzando su dominio de la lengua escrita, y en estos dos tipos de actividades prima la lectura y escritura del código escrito como único mediador del aprendizaje del currículo. A diferencia de estas prácticas bastante tradicionales, pudimos observar cómo cambia la participación de los mismos estudiantes cuando se los involucra en una dinámica diferente a la de la escritura individual.

Las estudiantes en práctica de pedagogía en este curso utilizaron en sus clases distintos tipos de materiales y dinámicas como un video explicativo, ejemplos en la pizarra en donde se invita a los estudiantes a participar, un mural y juegos interactivos sobre el contenido a enseñar. Con esta variedad de recursos, los estudiantes de $3^{\circ}$ básico se desplazan por el aula mientras participan en las actividades, aprovechan la disposición de los muebles para vincularse entre ellos y solucionar las tareas, se dirigen a las estudiantes de pedagogía para resolver dudas y se mantienen conectados a la actividad hasta su cierre.

Esta última práctica, permite a los estudiantes abrir su abanico de lo que significa aprender y ser aprendiz: no es solo rellenar guías escritas o transcribir al cuaderno lo que dice el profesor, sino también moverse por el aula y jugar, colaborar con los compañeros para solucionar tareas, pedir y recibir ayuda a los profesores.

Esto nos indica que, pese a la respuesta educativa institucionalizada de disponer el mobiliario en $\mathrm{U}$, por su potencial tanto para el trabajo colaborativo e innovación en las oportunidades de aprendizaje, no basta para cambiar las prácticas hacia unas más inclusivas. Son los estudiantes quienes aprovechan este potencial y las practicantes de pedagogía, aun no presionadas por la rendición de cuenta, quienes innovan en el aula. Mientras que los profesores pueden ignoran las conductas solidarias de los estudiantes o generar espacios de exclusión en ese espacio distinto, manteniendo estas como prácticas tradicionales.

\subsection{Educar cuando hay derechos vulnerados ¿Asistencialismo o inclusión?}

En ocasiones es difícil diferenciar cuando la educación en condición de vulnerabilidad responde con acciones desde un enfoque asistencialista o cuando es inclusivo. El asistencialismo se manifiesta como reacciones solidarias frente a situaciones que generan compasión ante las personas necesitadas, buscando responder a la desigualdad social mediante la provisión de elementos materiales básicos (Aguerrondo, 2008). El estudio de caso del cual rescatamos los ejemplos en este apartado, es el primer colegio, al cual asisten estudiantes de la zona, quienes habitan en tomas o lugares que no siempre tienen agua potable, alcantarillado ni electricidad, frecuentemente con padres y madres ausentes en el hogar por distintas razones. ¿Cómo distinguir cuando las acciones educativas ante la situación de vulnerabilidad buscan satisfacer un sentimiento de compasión o cuando son realizadas para promover y potenciar la participación y aprendizaje?

Con este foco destacamos algunas prácticas que aparentemente son asistencialistas, algunas de ellas institucionalizadas y otras como respuestas de algunos agentes educativos en particular.

Por ejemplo, cuando la escuela provee el uniforme deportivo a todos los estudiantes, ¿es asistencialismo? Dadas las condiciones de escaso poder adquisitivo de las familias, parece necesario apoyar con vestimenta financiada con dineros del Estado. Pero luego observamos que a veces los escolares vienen al colegio sin el uniforme, quienes los reciben en la puerta del colegio y de la sala de clase conversan con ellos -mi uniforme está mojado, 
lo lavé, llovió- y flexibilizan la exigencia de presentación personal para favorecer su participación en clases. Entonces comprendemos que hay una práctica consensuada entre los adultos de la escuela que conocen el entorno del escolar y eliminan una barrera: la norma de asistir a clases con uniforme.

Lo mismo ocurre con la provisión de los desayunos que, en general, se distribuyen en un determinado horario y lugar del colegio, sin embargo, muchos niños no llegan a esta hora o no pasan al comedor, por lo que sobran muchas porciones de colación, que por normativa se bota a la basura. En esta escuela durante los recreos y al término de la jornada, observamos que frecuentemente las manipuladoras de alimentos salen de las cocinas y comedores para instalarse con las bandejas en el patio, entregando panes o colaciones a todos los escolares. Entonces, nos preguntamos si esta es una práctica que solo apunta a resolver temporalmente una inequidad, o a una verdadera preocupación por alimentar a los niños para que puedan aprender en igualdad de condiciones, transformando los recreos de la escuela para ello.

También observamos algunas prácticas menos institucionalizadas, en las cuales un profesor jefe, por ejemplo, en un cajón de su escritorio cuenta con una serie de materiales escolares que él provee de manera personal, tales como lápices y gomas. Cuando comienza las actividades y observa que algunos de sus estudiantes no cuentan con sus útiles -se me quedó, mi mamá no me lo compró, se me acabó-, los incorpora rápidamente en la actividad entregándoles alguno de su cajón.

Lo mismo ocurre con un profesor de educación física, quien en sus bolsillos esconde yogurt, galletas, pan u otros, porque cuando reconoce que sus estudiantes no están en condiciones de participar en actividades que requieren de esfuerzo físico, comienza a entregar alimentos de su bolsillo, con lo que logra incorporarlos a la rutina de educación física. El manifiesta la importancia de tomar desayuno para su desarrollo, salud y rendimiento escolar, y conoce la realidad de sus estudiantes y familias.

Estas acciones realizadas por los actores educativos responden de manera concreta a la condición de vulnerabilidad de quienes se educan en este establecimiento. A veces estas prácticas se encuentran más institucionalizadas con la intención de disminuir las brechas en las condiciones en que los estudiantes enfrentan sus jornadas educativas, transformando algunos rituales en busca de promover un desarrollo integral de los alumnos, otras veces corresponden a la respuesta de un profesor en específico y su sensibilidad frente al reconocimiento de la desigualdad.

\section{Conclusiones}

En este artículo buscamos abordar de manera situada la complejidad de las distintas demandas que llegan a la escuela desde las políticas públicas, entendiendo las prácticas educativas como prácticas sociales en casos concretos de nuestra la educación pública.

La transformación de lo que ocurre en el aula, y en los espacios escolares en general, requiere de reconocer los esfuerzos de los distintos actores educativos para educar en contextos de alta vulnerabilidad, en los cuales se intenta dar sentido a la demanda de ser comunidad inclusiva en condiciones de pobreza y riesgo social. Intentar mejorar la escuela implica reconocer además que en las últimas décadas el foco en los aprendizajes de los estudiantes ha alcanzado dimensiones inauditas, instaurando sofisticados sistemas de evaluación nacional, implantándose el movimiento a favor de la rendición de cuentas 
(accountability) (Murillo y Krichesky, 2015). Los esfuerzos de las escuelas por destacar en cuanto al logro en lenguaje, matemáticas y ciencias juegan en otra dirección distinta de la inclusión (Redondo, 2005), cabe preguntase: qué efectos produce esta competencia en el trabajo colaborativo o cooperativo entre pares.

Muchas de las prácticas educativas observadas implican que la comunidad escolar o algunos de sus actores, flexibilizan lo que hacen para responder a las necesidades de su grupo de estudiantes, negociando estilos de regulación, usos de los espacios y muebles, formas de ofrecer los desayunos, en general, formas de legitimar/deslegitimar al aprendiz. Otras son iniciadas por los estudiantes de manera espontánea, sin que los adultos valoremos sus iniciativas para no excluir a sus compañeros, modelando una indiferencia colectiva (Slee, 2012).

Pero estas prácticas que destacamos ¿responden a un paradigma asistencialista o realmente nos encontramos ante intentos de inclusión? Algunas de estas prácticas responden a necesidades de manera momentánea, no necesariamente constituyen un cambio general o significativo en la condición de vulnerabilidad en la cuales los estudiantes viven diariamente, pero son parte del curriculum oculto: todo lo que la escuela enseña y los estudiantes aprenden cotidianamente. Los intersticios que aprovechan los actores educativos (Gómez-Hurtado, 2012), más allá de los profesores de estos dos estudios de caso, para dar prioridad a la solidaridad y valores inclusivos se abren paso en un medio que a la vez los incentiva a la competencia. En este contexto de contradicciones enseñan como convivir, con otras herramientas intelectuales, afectivas y sociales (Rivero, 2013), recobrando el sentido formativo de la escuela.

Sin duda, para que este proyecto político avance se requieren cambios importantes en las creencias de quienes conforman y rodean las escuelas y la sociedad en general, para entre todos pensar en cómo favorecer el desarrollo de todas las personas en la sociedad, más allá de las fronteras de la escuela y a pesar de las contradicciones que vive la sociedad chilena actual. La inclusión hoy en día en Chile constituye un desafío, con un compromiso especial con la educación pública, la cual concentra la mayoría de los estudiantes y donde las condiciones desiguales actuales son un obstáculo mayor para educar y ofrecer un espacio desafiante y atractivo para el desarrollo de las personas.

Es así que la educación inclusiva constituye "un proyecto político en el que tratamos de identificar las formas complejas en que los obstáculos impiden que los estudiantes accedan, participen realmente y tengan éxito en la educación” (Slee, 2012, p. 124), es a este proceso político al cual los profesores investigadores intentamos aportar.

\section{Agradecimientos}

Esta investigación ha sido parcialmente financiada por PIA- CONICYT CIE 160009.

\section{Referencias}

Achilli, E. (2013). Investigación socioantropológica en educación. Para pensar la noción de contexto. En N. E. Elichiry (Comp.), Historia y vida cotidiana en educación (pp. 33-48). Buenos Aires: Ed. Manantial.

Aguerrondo, I. (2008). Revisar el modelo: Un desafío para lograr la inclusión. Perspectiva, 28(1), 61 80. 
Artiles, A. y Kozleski, E. (2016). Inclusive education's promises and trajectories: Critical notes about future research on a venerable idea. Education Policy Analysis Archives, 24(43), 1-24. https://doi.org/10.14507/epaa.24.1919

Ainscow, M. (2005). La mejora de la escuela inclusiva. Cuadernos de Pedagogía, 349, 78-83.

Ainscow, M. y Booth, T. (2015). Guía para la educación inclusiva: Desarrollando el aprendizaje y la participación en los centros escolares. Madrid: OEI.

Awasthy, R. (2015). Journey of doing quasi-ethnographic study in organizations. Sage journals, 19(3), 219-226. https://doi.org/10.1177/0972262915593667

Baquero, R. (2013). Sospechas cruzadas. El problema de la educabilidad. Diálogos pedagógicos, 11(21), 157-190.

Ball, S. (2001). Performativities and fabrications in the education economy: Towards the performative society. En D. Gleeson y C. Husbands (Eds.), The performing school: Managing teaching and learning in a performance culture (pp. 210-226). Londres: Routledge.

Bascuñan, N., Contreras, X., Herrera, J., Saavedra, C. y Valdebenito, J. (2017). Prácticas y teorías implícitas respecto a la respuesta educativa frente a la diversidad presente en dos $3^{\circ}$ años básicos de colegios municipales de la ciudad de Viña del Mar. Trabajo Fin de Grado. Pontificia Universidad Católica de Valparaíso, Valparaíso.

Booth, T. y Ainscow, M. (2002). Guía para la evaluación y mejora de la educación inclusiva. Desarrollando el aprendizaje y la participación en las escuelas. Madrid: Consorcio Universitario para la Educación Inclusiva.

Castro, R. (2017). Revisión y análisis documental para estado del arte: Imaginarios sociales sobre inclusión educativa. Revista Latinoamericana de Inclusión Educativa, 11(1), 283-297.

Dávila, O., Ghiardo, F. y Medrano, C. (2005). Los desheredados. Trayectorias de vida y nuevas condiciones juveniles. Valparaíso: Ediciones CIDPA.

Duschatzky, S. y Birgin, A. (2001). ¿Dónde está la escuela? Buenos Aires: Ediciones Manantial SRL.

Echeita, G., Muñoz, Y., Sandoval, M. y Simón, C. (2014). Reflexionando en voz alta sobre el sentido y algunos saberes proporcionados por la investigación en el ámbito de la educación inclusiva. Revista Latinoamericana de Educación Inclusiva, 8(2), 25-48.

Escudero, J. y Martínez, B. (2012). Las políticas de lucha contra el fracaso escolar: ¿Programas especiales o cambios profundos del sistema y la educación? Revista de Educación, número extraordinario, 174-193.

Godoy, G y Córdova, J. (2017). Significados ocultos: Pretendiendo ser objetivo. En D. Manghi (Ed.). La complejidad de la interacción en el aula: Reconociendo significados que transforman (pp. 65-81). Valparaíso: EUV.

Gómez-Hurtado, I. (2012). Una dirección escolar para la inclusión escolar. Perspectivas Educacionales, 51(2), 21-45.

Graham, L. y Slee, R. (2008). An illusory interiority: Interrogating the discourse/s of inclusion. Educational Philosophy and Theory, 40(2), 277-292. https://doi.org/10.1111/j.14695812.2007.00331.x

Grech, S. (2017, agosto). Inclusión y participación desde una mirada crítica. Conferencia presentada en el Seminario Miradas Críticas de la Inclusión y Participación en el Abordaje de las Necesidades Educativas Especiales. Pontificia Universidad Católica de Valparaíso, Chile. 
Infante, M., Matus, C., Paulsen, A., Salazar, A. y Vizcarra, R. (2013). Narrando la vulnerabilidad escolar: Performatividad, espacio y territorio. Literatura y Lingüística, 27, 281-308. https://doi.org/10.4067/SO716-58112013000100014

Jorquera, C., Gunther, L., González, R. y Martínez, E. (2017). Enseñar en la otra Viña del Mar. En D. Manghi. (Ed.). La complejidad de la interacción en el aula, reconociendo significados que transforman (pp. 19-23). Valparaíso: Ediciones Universitarias de Valparaíso.

Julio, C. (2017). Configurando identidad de aprendiz en la escuela: Obstáculos en la relación pedagógica y su indicendia en procesos de “deserción” escolar temprana. Revista Latinoamericana de Inclusión Educativa, 11(1), 109-129. https://doi.org/10.4067/So71873782017000100008

Julio, C., Conejeros, L., Rojas, C., Mohammad, M., Rubí, Y. y Cortés, A. (2016). Desencuentro cultural en el aula. Una barrera al aprendizaje de niños y niñas en situación de pobreza. Revista Mexicana de Investigación Educativa, 21(68), 71-94.

Lave, J. y Wenger, E. (1991). Situated learning legitimate peripheral participation. Nueva York, NY: Cambridge University Press. https://doi.org/10.1017/CBO9780511815355

López, V., Julio, C., Morales, M. y Pérez, M. (2012). Barreras culturales para la inclusión: Políticas y prácticas de integración en Chile. Revista de Educación, 363, 256-281.

Manghi, D. (Ed.). (2017). La complejidad de la interacción en el aula, reconociendo significados que transforman. Valparaíso: Ediciones Universitarias de Valparaíso.

Manghi D. y Aranda, I. (2017, noviembre). Resemiotizando la inclusión: Análisis multimodal de dos ediciones del index. Ponencia presentada en el XVIII Congreso Alsfal: Diálogos entre saberes, enfoques y prácticas. Universidad Nacional de Córdoba, Argentina.

Manghi, D. y Baeza, P. (2017,octubre). Construcción discursiva multimodal de la noción de inclusión educativa en documentos de política pública. Ponencia presentada en el XII Congreso Internacional de la Asociación Latinoamericana de estudios del discurso. Discurso, construcción y transformación social. Pontificia Universidad de Chile, Chile.

MINEDUC. (2014). Programa bibliotecas escolares CRA 2010-2014. Recuperado de http://www.cultura.gob.cl/wp-content/uploads/2014/08/recuento-cra-2O-FOCO20102014.pdf

MINEDUC. (2016). Orientaciones para la construcción de comunidades educativas inclusivas. Recuperado de https://media.mineduc.cl/wpcontent/uploads/sites/28/2017/07/Orientaciones-Comunidades-EducativasInclusivas.pdf

Mizala, A. y Torche, F. (2012). Bringing the schools back in: The stratification of educational achievement in the Chilean voucher system. International Journal of Educational Development, 32(1), 132-144. https://doi.org/10.1016/j.ijedudev.2010.09.004

Moreno-Doña, A. y Gamboa, R. (2014). Dictadura chilena y sistema escolar: A otros dieron de verdades a cosa llamada educación. Educar em Revista, 51, 51-66. https://doi.org/10.1590/S0104-40602014000100005

Murillo, F. (2003). El movimiento teórico práctico de mejora de la escuela. Algunas lecciones aprendidas para transformar los centros docentes. REICE. Revista Iberoamericana sobre Calidad, Eficacia y Cambio en Educación, 1(2), 1-22.

Murillo, F. y Krichesky, G. (2015). Mejora de la escuela: Medio siglo de lecciones aprendidas. REICE. Revista Iberoamericana sobre Calidad, Eficacia y Cambio en Educación, 13(1), 69-102. 
OECD. (2004). Revisión de políticas nacionales de educación. París: OECD. https://doi.org/10.1787/9789264021020-es

OECD. (2012). Equity and quality in education: Supporting disadvantaged students and schools. París: OECD.

OECD. (2017). Revisión de recursos escolares: Chile 2017. París: OECD.

Opazo, C. (2013, agosto). Educación inclusiva y pedagogía en contextos de desventaja socioeducativa. Ponencia en el las primeras Jornadas de Jóvenes Investigadores en Educación. FLACSO, Buenos Aires, Argentina.

Raczynski, D. y Muñoz, G. (2007). Reforma educacional chilena: El difícil equilibrio entre la macro y la micropolítica. Santiago: CIEPLAN.

Raczynski, D., Muñoz, G., Weinstein, J. y Pascual, J. (2013). Subvención escolar preferencial (SEP) en chile: un intento por equilibrar la macro y micro política escolar. REICE. Revista Iberoamericana sobre Calidad, Eficacia y Cambio en Educación, 11(2), 164-193.

Redondo, J. (2005). El experimento chileno en educación ¿Conduce a mayor equidad y calidad en la educación? Última Década, 22, 95-110.

Rivero, R. (2013). Educación y pedagogía en el marco del neoliberalismo y la globalización. Perfiles Educativos, 35(142), 149-164. https://doi.org/10.1016/So185-2698(13)71854-3

Sinisi, L. (2013). Contribuciones de la etnografía para el estudio de redes y tramas psicoeducativas. En N. E. Elichiry (Comp.), Historia y vida cotidiana en educación (pp. 49-66). Buenos Aires: Ed. Manantial.

Slee, R. (2012). La escuela extraordinaria: exclusión, escolarización y educación inclusiva. Madrid: Morata.

Slee, R. (2013). How do we make inclusive education happen when exclusion is a political predisposition? International Journal of Inclusive Education, 17(8), 895-907. https://doi.org/10.1080/13603116.2011.602534

Slee, R. y Allan, J. (2011). Excluding the included: A reconsideration of inclusive education. International Studies in Sociology of Education, 11(2), 173-192. https://doi.org/10.1080/09620210100200073

Strauss, A. y Corbin, J. (2002). Bases de la investigación cualitativa. Técnicas y procedimientos para desarrollar la Teoría Fundamentada. Medellín: Universidad de Antioquia.

Terigi, F. (2009). El fracaso escolar desde la perspectiva psicoeducativa: Hacia una reconceptualización situacional. Revista Iberoamericana de Educación, 20, 23-39.

UNESCO. (1990). Declaración mundial sobre educación para todos y marco de acción para satisfacer las necesidad básicas de aprendizaje. Jomtien: UNESCO.

UNESCO. (1994). Declaración de Salamanca y marco de acción para las necesidades educativas especiales. Salamanca: UNESCO.

Weinstein, J., Fuenzalida, A. y Muñoz, G. (2010). La subvención preferencial: Desde una difícil instalación hacia su institucionalización. En S. Martinic y G. Elacqua (Eds.), ¿̨Fin de ciclo? Cambios en la gobernanza del sistema educativo, (pp. 161-182). Santiago de Chile: Pontificia Universidad Católica de Chile. 


\section{Breve CV de las autoras}

\section{Dominique Manghi}

Licenciada en Educación y Doctora en Lingüística. Actualmente es profesora adjunta de la Escuela de Pedagogía de la Pontificia Universidad Católica de Valparaíso, e investigadora principal del Centro de Investigación para la Educación Inclusiva, a cargo de la línea 4: Practicas de Aula y de Escuela para la Inclusión (PIA-CONICYT CIE160009). Formadora de profesores e investigadora en análisis de la interacción escolar, análisis del discurso, multimodalidad, alfabetización y Semiótica Social. Ha participado en varias investigaciones y dirigido los Fondecyt 11100169 (2010-2012), Fondecyt 1130684.(2013-2015) y Fondecyt 1180472 (2018-2020). Editora y coautora del libro: La complejidad de la interacción en el aula: reconociendo significados que transforman (2017). ORCID ID: https://orcid.org/O000-0002-0278-9899. Email: dominique.manghi@pucv.cl

\section{Camila Saavedra}

Licenciada en Educación y Profesora de Educación Diferencial con Mención en Trastornos de Aprendizaje Específico, Pontificia Universidad Católica de Valparaíso. Estancia de un semestre en la Universidad de Murcia, España. Actualmente se desempeña como maestra de educación especial en el departamento psicopedagógico del Instituto Jean Piaget en Tultepec, México. ORCID ID: https://orcid.org/O000-0002-0191-9516 Email: camila.saavf@gmail.com

\section{Nicoley Bascuñan}

Licenciada en Educación y Profesora de Educación Diferencial con mención en Retardo Mental de la Pontificia Universidad Católica de Valparaíso. Se ha desarrollado como Directora del centro de Atención para hijos de madres Temporeras en la comuna de Cabildo, Chile. Actualmente trabaja dentro del Programa Integración Escolar del Liceo Polivalente $\mathrm{A}^{\circ} 2$ de la comuna de Cabildo. ORCID ID: https://orcid.org/0000-00025616-6515 Email: nicoleybascu@gmail.com 